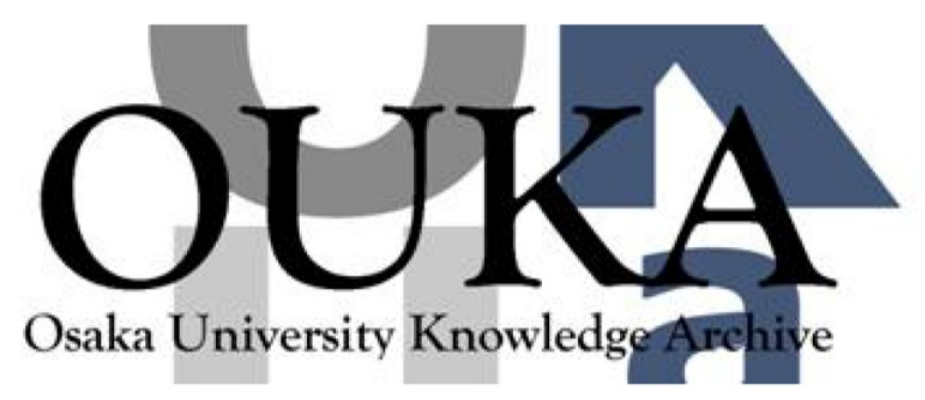

\begin{tabular}{|c|l|}
\hline Title & $\begin{array}{l}\text { Preparation of Long Conjugated Porphyrin } \\
\text { Polymers with Gold Nanoparticles at Both Ends } \\
\text { as Electronic and/or Photonic Molecular Wires }\end{array}$ \\
\hline Author(s) & $\begin{array}{l}\text { Ozawa, Hiroaki; Kawao, Masahiro; Tanaka, } \\
\text { Hirofumi et al. }\end{array}$ \\
\hline Citation & CHEMISTRY LETTERS. 38(6) p. 542-p.543 \\
\hline Issue Date & $2009-06-05$ \\
\hline oaire:version & VoR \\
\hline URL & https://hdl.handle. net/11094/3261 \\
\hline rights & \\
\hline Note & \\
\hline
\end{tabular}

Osaka University Knowledge Archive : OUKA

https://ir. Library. osaka-u. ac. jp/

Osaka University 


\title{
Preparation of Long Conjugated Porphyrin Polymers with Gold Nanoparticles at Both Ends as Electronic and/or Photonic Molecular Wires
}

\author{
Hiroaki Ozawa, ${ }^{1}$ Masahiro Kawao, ${ }^{2}$ Hirofumi Tanaka, ${ }^{3}$ and Takuji Ogawa*2,3 \\ ${ }^{1}$ Graduate School of Engineering, Nagoya University, Furo-cho, Chikusa-ku, Nagoya 464-8603 \\ ${ }^{2}$ Research Center for Molecular-Scale Nanoscience, Institute for Molecular Science, \\ 5-1 Higashiyama, Myodaiji, Okazaki 444-8787 \\ ${ }^{3}$ Graduate School of Science, Osaka University, 1-1 Machikaneyama-cho, Toyonaka 560-0043
}

(Received March 5, 2009; CL-090225; E-mail: hiro.ozawa@mail.cstm.kyushu-u.ac.jp)

\begin{abstract}
Nanocomposites of long conjugated porphyrin polymers with both ends substituted by gold nanoparticles were prepared by mixing a polymer bearing thiol groups at both ends with gold nanoparticles protected by 2-methylundecane-2-thiol. The nanocomposites were observed by atomic force microscopy (AFM), which showed that the gold nanoparticles were attached at the terminal positions of the porphyrin polymers as expected from height analyses. The height at the terminal position was ca. 3$4 \mathrm{~nm}$ and that at the center position was ca. $2 \mathrm{~nm}$. The nanocomposites could be dispersed homogeneously on solid surfaces such as HOPG or glass substrates using a pulse injection method under high vacuum.
\end{abstract}

Gold nanoparticles connected by conjugated $\pi$-electronic systems have attracted much attention as promising materials for electronics, ${ }^{1,2}$ photonics, ${ }^{3,4}$ nanomechanics, ${ }^{5}$ molecular spintronics, ${ }^{6}$ sensor applications, ${ }^{7}$ and photovoltaic cells. ${ }^{8}$ We are interested in the preparation of gold nanoparticles/long conjugated $\pi$-electronic systems as electronic and photonic molecular wires. In a previous paper we reported the preparation of a one-dimensional array of gold nanoparticles bonded to porphyrin units of a conjugated porphyrin polymer, and found that energy transfer occurred between the gold nanoparticles and the porphyrin polymer. ${ }^{9}$ In order to elucidate the interaction of the plasmon excitation of gold nanoparticles with the conjugated porphyrin polymer, we studied porphyrin polymers bearing gold nanoparticles at the terminal positions using time-resolved scanning near field optical microscopy (SNOM). ${ }^{10}$ Using these measurements we hope to develop a molecular photonic wire. Photonic wires require that the distance between the gold nanoparticles be more than $50 \mathrm{~nm}$. To our knowledge, there are no reports of gold nanoparticle composites connected by such long conjugated molecules. Here we report the preparation of a composite that fulfills these requirements, and measurements of some preliminary optical properties.

Previously, we reported the synthesis of end-functionalized conjugated porphyrin oligomers (monomer to heptamer) and studied their optical properties. ${ }^{11}$ Here we used a similar procedure and isolated longer homologues (degree of polymerization $n=$ ca. 15-200) by recycling preparative GPC-HPLC. The molecular weight distribution of the isolated mixture $\mathbf{1}$ was determined by analytical GPC using polystyrene standards (Figure S1). ${ }^{12}$ The ${ }^{1} \mathrm{HNMR}$ data on 1 were presented in the Supporting Information. ${ }^{12}$ Gold nanoparticles were prepared using 2-methylundecane-2-thiol as the protecting molecule instead of $n$-dodecane-1-thiol, originally reported by Brust et al. ${ }^{13}$ The diameter of the nanoparticles $\mathbf{2}$ observed by transparent electron

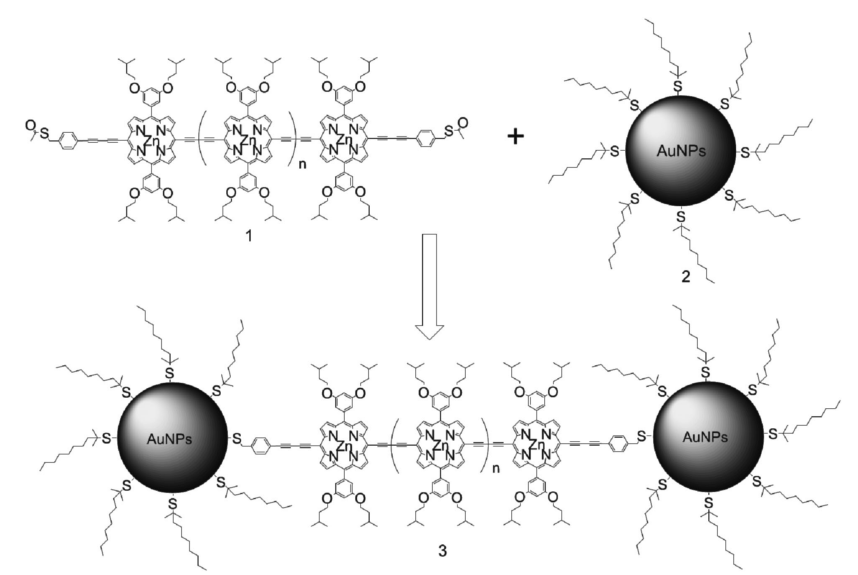

Scheme 1. Preparations of gold nanoparticle terminated porphyrin polymer 3 .

microscopy was $2.4 \pm 0.8 \mathrm{~nm}$. Gold nanoparticle-terminated porphyrin polymers 3 were prepared by mixing these two components (Scheme 1). Details of these preparation methods are described in the Supporting Information. ${ }^{12}$

As shown in Figure 1a, the UV-vis absorption spectrum of $\mathbf{3}$ was nearly identical to superimposed spectra of $\mathbf{1}$ and $\mathbf{2}$, indicating the absence of any significant electronic interaction between the nanoparticles and the porphyrin polymer in the ground state. The fluorescence spectrum of $\mathbf{3}$ (Figure 1b) exhibited an emission peak at $834 \mathrm{~nm}$ that was also identical with that of $\mathbf{1}$. In the case of a porphyrin polymer having gold nanoparticles at every fourth porphyrin unit (4, Figure S2), ${ }^{12}$ the fluorescence spectrum was perturbed and partially quenched. ${ }^{9}$ The most probable reason for the absence of interactions at ground and excited states in $\mathbf{3}$ is the small ratio of gold nanoparticles/porphyrin units. Even if there is some interaction between the nanoparticles and the porphyrin in $\mathbf{3}$, the ratio of nanoparticles to the porphyrin units $(\approx 2 / 50)$ may make it too small to be observed.

The structure of nanocomposite $\mathbf{3}$ was examined by atomic force microscopy (AFM) on surfaces of mica, HOPG and modified glass. A solution of $\mathbf{3}$ in THF (the absorbance at $450 \mathrm{~nm}$ was set to 0.1 ) was cast on mica and dried. The surface was observed by tapping mode AFM under ambient conditions. A typical image over a wide range $\left(2.5 \times 2.5 \mu \mathrm{m}^{2}\right)$ is shown in Figure $2 \mathrm{a}$ together with a picture at higher magnification $\left(250 \times 250 \mathrm{~nm}^{2}\right)$ in Figure 2b. In Figure 2a, many pairs of nanoparticles together with small amounts of trimers, tetramers, or higher nanoparticles can be seen. In the magnified image a string that connects two nanoparticles is clearly observed. The heights of the terminal nanoparticles are ca. $3-4 \mathrm{~nm}$ and that of the string is ca. $2 \mathrm{~nm}$ 

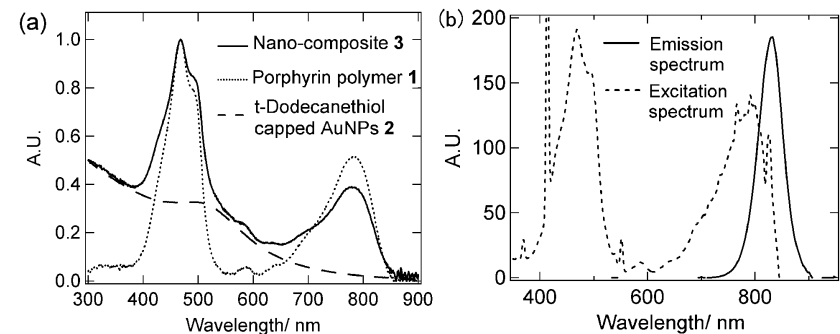

Figure 1. (a) UV-vis absorption spectra of gold nanoparticleterminated porphyrin polymer $\mathbf{3}$ in THF (solid line), porphyrin polymer 1 (dotted line), and 2-methylundecane-2-thiol-capped gold nanoparticles 2 (broken line). (b) Emission (solid line) and excitation (broken line) spectra of $\mathbf{3}$.
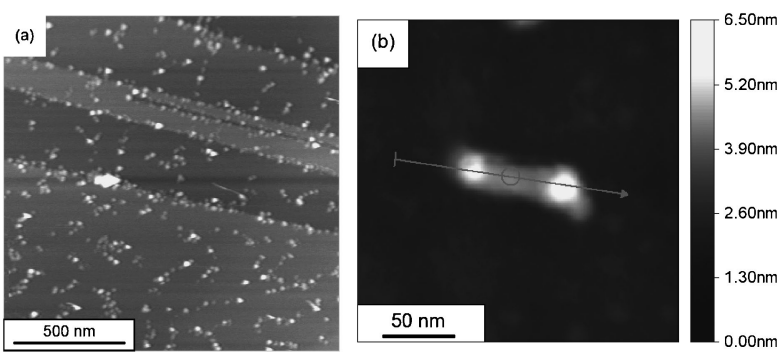

(c)

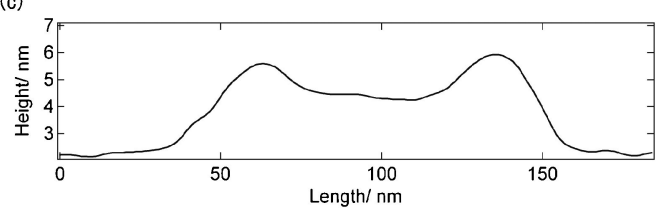

Figure 2. (a) AFM image of gold nanoparticle-terminated porphyrin polymers on HOPG substrates prepared by the cast method. (b) Magnified images and (c) cross-section of nanocomposite on mica substrate. Arrow represents cross-section.

(Figure 2c). Estimating from the gold core of the nanoparticles observed by TEM $(2.4 \pm 0.8 \mathrm{~nm})$ and the length of the protecting alkyl thiol (ca. $1.4 \mathrm{~nm}$ ), 3-4 nm is a reasonable size for the gold nanoparticles. The diameter of the porphyrin polymer simulated by MM2 calculations is $2.3 \mathrm{~nm}$, which is also consistent with the height of the string. Thus, it is rational to conclude that the nanocomposites observed by AFM are gold nanoparticles connected by the porphyrin polymer molecule.

In order to study molecular scale photonic and electronic properties, these nanocomposites must be dispersed on transparent and/or insulating surfaces such as glass. However, the cast deposition on glass surface did not disperse the nanocomposite homogeneously, because the glass surface is hydrophilic and the nanocomposite is hydrophobic. For homogeneous dispersion, we used a combination of a modified glass surface and the pulse injection method. ${ }^{14}$ Glass substrates were treated with trimethoxy( $N$-phenyl-3-aminopropyl)silane to make the surface hydrophobic and increase affinity toward the nanocomposite. ${ }^{9}$

The substrates (the surface modified glass or HOPG) were placed in a vacuum chamber, and a tetrahydrofuran (THF) solution of the gold nanoparticle-terminated porphyrin polymers (ABS = ca. 0.07 at $468 \mathrm{~nm}$ ) was sprayed on the substrates through a pulse injection valve for about $10 \mu$ s into the chamber. After spraying the nanocomposite on the substrates, the surfaces

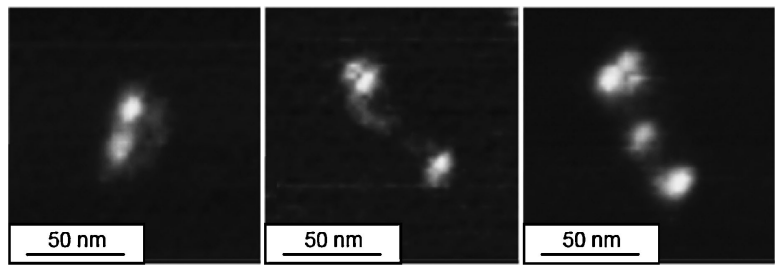

Figure 3. AFM images of typical nanoparticle porphyrin composites. Left: Two particles are connected by a curved porphyrin polymer. Center: Two particles are connected by a twisted porphyrin polymer. Right: Three or four particles are connected.

were observed by AFM (Figure S3a for HOPG and Figure S3b for the modified glass). ${ }^{12}$ The nanocomposites could be deposited more homogeneously with fewer aggregates on the surfaces using this method than the cast method.

The distribution of distances between the gold nanoparticles in the composites is shown in Figure S4. ${ }^{12}$ The distribution is centered around $40 \mathrm{~nm}$ which is significantly shorter than the molecular length $(76 \mathrm{~nm})$ estimated from the GPC analysis of the porphyrin polymer. This is explained by the observation that long molecules, even though they are bonded only by $\mathrm{sp}^{2}$ and sp hybridized carbon, tend to be curved and twisted on solid surfaces as shown in Figure 3. ${ }^{9}$

Gold nanoparticle-terminated porphyrin polymers were successfully fabricated from thiol-substituted porphyrin polymers and 2-methylundecane-2-thiol-capped gold nanoparticles as confirmed by AFM observation. The nanocomposites could be homogeneously deposited on HOPG and chemically modified glass surfaces using a pulse injection method. Studies of electric and optical properties of the gold nanoparticle-terminated porphyrin polymers on solid surfaces are in progress.

This work was supported by a Grant-in-Aid for Scientific Research (No. 19310079) from the Ministry of Education, Culture, Sports, Science and Technology of Japan.

\section{References and Notes}

1 M. A. Reed, T. Lee, Molecular Nanoelectronics, American Scientific Publishers, Los Angeles, California, 2003.

2 M. Brust, D. J. Schiffrin, D. Bethell, C. J. Kiely, Adv. Mater. 1995, 7, 795.

3 T. Tozawa, Chem. Commun. 2004, 1904.

4 G. P. Wiederrecht, G. A. Wurtz, J. Hranisavljevic, Nano Lett. 2004, 4, 2121.

5 R. A. van Delden, M. K. J. ter Wiel, M. M. Pollard, J. Vicario, N. Koumura, B. L. Feringa, Nature 2005, 437, 1337.

6 T. Sugawara, M. Minamoto, M. M. Matsushita, P. Nickels, S. Komiyama, Phys. Rev. B 2008, 77, 235316.

7 E. Katz, I. Willner, Angew. Chem., Int. Ed. 2004, 43, 6042.

8 H. Imahori, A. Fujimoto, S. Kang, H. Hotta, K. Yoshida, T. Umeyama, Y. Matano, S. Isoda, Adv. Mater. 2005, 17, 1727.

9 H. Ozawa, M. Kawao, H. Tanaka, T. Ogawa, Langmuir 2007, 23, 6365.

10 K. Imura, T. Nagahara, H. Okamoto, J. Am. Chem. Soc. 2004, 126, 12730.

11 H. Ozawa, M. Kawao, H. Tanaka, T. Ogawa, Tetrahedron 2006, 62, 4749.

12 Supporting Information is available electronically on the CSJ-Journal Web site, http://www.csj.jp/journals/chem-lett/index.html.

13 M. Brust, M. Walker, D. Bethell, D. J. Schiffrin, R. Whyman, J. Chem. Soc., Chem. Commun. 1994, 801.

14 H. Tanaka, C. Hamai, T. Kanno, T. Kawai, Surf. Sci. 1999, 432, L611. 